\title{
The effect of voluntary lateral trunk bending on balance recovery following multi-directional stance perturbations
}

\author{
U. M. Küng • C. G. C. Horlings · F. Honegger • \\ J. H. J. Allum
}

Received: 10 September 2009 / Accepted: 5 February 2010 / Published online: 4 March 2010

(C) Springer-Verlag 2010

\begin{abstract}
Stabilising shifts of the centre of mass (COM) are observed during balance recovery when subjects simultaneously execute voluntary unilateral knee flexion or unilateral arm raising. Here, we examined whether voluntary lateral trunk bending provided more beneficial stabilising effects, and how motor programs of balance corrections are combined with those of the focal voluntary action. The upright balance of 24 healthy young subjects (19-33 years of age) was perturbed using multi-directional rotations of the support-surface. The perturbations consisted of combined pitch and roll rotations $\left(7.5^{\circ}\right.$ and $\left.60^{\circ} / \mathrm{s}\right)$ presented randomly in six different directions. Three conditions were tested: perturbation of stance only (PO); combined balance perturbation and cued uphill bending of the trunk (CONT); and combined perturbation and cued downhill bending of the trunk (IPS). For comparison, subjects were required to perform trunk bending alone (TO). Outcome measures were biomechanical responses and surface EMG activity of several muscles. Calculated predicted outcomes (PO + TO) were compared with combined measures (CONT or IPS). CONT trunk bending uphill showed two phases of benefit in balance recovery for laterally but, in contrast to voluntary knee bending, not for posterior directed components of the perturbations. IPS trunk bending had negative effects on balance. Early balance correcting muscle responses were
\end{abstract}

U. M. Küng · C. G. C. Horlings · F. Honegger · J. H. J. Allum ( $₫)$ Department of ORL, University ORL Clinic,

University Hospital, Petersgraben 4,

4031 Basel, Switzerland

e-mail: jallum@uhbs.ch

C. G. C. Horlings

Department of Neurology,

Radboud University Medical Centre,

Nijmegen, The Netherlands marginally greater than PO responses. Prominent secondary balance correcting responses, having a similar timing as voluntary responses observed under TO conditions, were seen under CONT only in trunk muscles. These, and later stabilising, responses had amplitudes as expected from $\mathrm{PO}+\mathrm{TO}$ conditions being significantly greater than $\mathrm{PO}$ responses. The ability with which different muscle synergies for balance corrections and voluntary trunk bending were integrated into one indicates a flexible adjustment of the CNS programs to the demands of both tasks.

Keywords Balance corrections · Postural control . Muscle responses $\cdot$ CNS motor programs

\section{Introduction}

A new body equilibrium can be achieved in an anticipatory mode, for example, by accommodating body lean on approach to a natural slope of the ground. A change in posture can also occur in a feedback mode following a perturbation to balance. If the muscle synergies underlying these two modes of control-anticipatory and feedback-are very similar, as several authors have suggested (Hughey and Fung 2005; Nashner and Cordo 1981; Pozzo et al. 2001), then presumably training anticipatory motor control should improve balance and help reduce falls when a perturbation occurs (Marigold et al. 2005).

Similarity of the muscle synergies in anticipatory and feedback modes could imply: firstly, that anticipatory postural adjustments (APA) present prior to main voluntary actions have amplitude and timing characteristics similar to those of early automatic postural reactions (APRs) elicited during balance corrections and any preceding stretch reflex activity. Secondly, that the muscle activity underlying the 
voluntary action and later balance stabilising activity after APRs also have similar characteristics. When the first condition is met, it has been proposed that the CNS can easily incorporate the two types of muscle synergies into one (Küng et al. 2009b). The studies of Küng et al. (2009b) demonstrated that when voluntary unilateral knee bending and balance correcting postural responses to tilt perturbations were combined these were well integrated together, because timing and excitation levels of stretch reflex activity, APRs and APAs were synchronised. In contrast, combining bilateral voluntary knee bending with balance corrections was not effective because responses to backward tilt produced inhibitory reflex responses in agonistic tibialis anterior muscles of both legs when excitatory APA activity would normally occur (Oude Nijhuis et al. 2007). The result was instability of the centre of mass (COM). Consistent with this instability, APRs and later stabilising activity was generally greater than predicted (Oude Nijhuis et al. 2007). That is the muscle action when voluntary and perturbation tasks were combined was greater than for the separately performed voluntary and perturbation tasks. In contrast, the amplitudes of later stabilising activity were less than predicted for the more stable unilateral knee bending paradigm (Küng et al. 2009b). These results on knee responses indicate that voluntary activity can be easily integrated with balance corrections when APAs and APR with preceding reflex activity are similar in timing and direction of excitation but that later voluntary and stabilising action could be dissimilar and yield combined activity patterns with lower amplitudes than predicted.

Movements of other body segments such as the arms and trunk also play a major role in aiding acquisition of a new equilibrium when stance is perturbed (Küng et al. 2009a). The question arises whether adding voluntary action by the arms or trunk would aid stability in a similar manner as unilateral knee bending. Arm raising uphill lead to similar improvements in lateral COM displacements as unilateral knee bending but no improvement in anterior-posterior (AP) COM displacements (Grin et al. 2007). The lack of AP action in contrast to voluntary unilateral knee bending is not unexpected given the combined roll and pitch action of knee muscles (Küng et al. 2009c). Interestingly, in contrast to AP arm raising (Friedli et al. 1984), little changes in leg muscle activity were observed with arm abduction and there was a general lack of APA in the trunk muscles. Furthermore, the arm action required greater muscle activity than predicted in trunk muscles possibly due to simultaneous counter rotation of the trunk.

It is not known if voluntary movements of trunk initiated when cued by the onset of a balance correction also aid stability. From the results described above, we concluded that synergy conditions similar to those present with unilateral knee bending need to be met if voluntary trunk bending would be beneficial to COM stability following tilt perturbations. Our hypothesis was that the presence of APA associated with this voluntary action would aid the CNS to release an appropriate combined response that would be a well-integrated combination of anticipatory and balance correcting synergies. Thereby, less muscle activity than predicted would be required to achieve a later stabilising action. If we could show that not only unilateral uphill knee bending but also voluntary trunk bending uphill have beneficial effects for balance stability when stance is perturbed, then our results would have a general applicability for rehabilitation programs.

From a scientific viewpoint, the question arises how anticipatory and feedback strategies are combined when the voluntary movement involves a much larger mass such as the trunk. Biomechanically, voluntary movements of the trunk can be expected to be executed more slowly than those of unilateral knee bending with associated changes in underlying muscle synergies. Thus, we posed the following questions: First, can standing subjects implement voluntary lateral trunk flexion movements simultaneously when their balance is being perturbed by support-surface tilt and is the effect on COM displacement positive or negative? Second, would incorporation of voluntary synergy into the early balance correcting responses and later stabilising responses alter the form and amplitudes of EMG responses in trunk muscles? That is, we investigated whether the muscle and subsequent biomechanical responses could be predicted from the individual responses for voluntary trunk bending and in response to the balance perturbations. Third, what common roles might underly APAs prior to the main voluntary activity, APRs and preceding reflex responses prior to balance corrections, in shaping the individual and the combined synergies?

\section{Materials and methods}

\section{Subjects}

Twenty-four healthy young subjects (mean \pm SEM: age $25 \pm 0.8$; height $173 \pm 1.5 \mathrm{~cm}$; and body mass $62 \pm 1.7 \mathrm{~kg}$ ) without neurologic or orthopaedic deficits participated in the experiments. All subjects gave witnessed, written informed consent to participate in the experiments according to the Declaration of Helsinki. The Institutional Ethical Review Board of the University Hospital of Basel approved the study.

\section{Protocol}

Subjects stood on a servo-controlled platform that could tilt in the pitch and roll directions. The roll and the pitch axis of 
the platform had the same height and passed between the feet. The stance width was standardised (14-cm foot separation). Subjects were informed that they were allowed to grasp two handrails of adjustable height mounted on either side of the platform if they needed support. The subjects' feet were lightly strapped across the instep, and heel guides fixed to the upper surface of the support-surface prevented backwards movement of the feet. The heel guides were adjusted to ensure that the ankle joint axes were aligned with the pitch axis of the platform and, with the instep straps, prevented stepping reactions when platform tilts occurred. One assistant was present to lend support in case of a fall, but no falls, and just four near falls (defined as a need to grasp the handrail or receive assistance) occurred for backward tilts of the support-surface when simultaneous voluntary bending of the trunk downhill was required.

The test protocol was identical to that of Grin et al. (2007) and Küng et al. (2009b). Stimuli consisted of rotations of the platform in 6 different directions with a constant velocity of $60^{\circ} \% \mathrm{~s}$ and a constant amplitude of $7.5^{\circ}$. Perturbation directions were combinations of pitch and roll rotations, where $0^{\circ}$ was defined as a pure-pitch perturbation, toes down, $90^{\circ}$ as a pure right tilt, $180^{\circ}$ a pure-pitch toe-up perturbation. The combinations used were forward right (direction $45^{\circ}$ ) and forward left $\left(315^{\circ}\right)$ together termed anterior-lateral or $\mathrm{AL}$, right backward $\left(113^{\circ}\right)$ and left backward $\left(248^{\circ}\right)$, collectively termed, lateral-posterior or LPo, and backward right $\left(158^{\circ}\right)$ and backward left $\left(203^{\circ}\right)$ and termed posterior and lateral (PoL). A schematic of the tilt directions is shown in Fig. 1. Each perturbation direction was presented 7 times in random order to the subject for each task condition except for one additional PoL perturbation which was presented first and therefore 8 times in total. Perturbation task conditions were support-surface rotation only (PO), combined support-surface rotation and cued voluntary lateral bending of the trunk, contralateral to tilt (CONT), and combined support-surface rotation and cued lateral bending of the trunk, ipsilateral to tilt (IPS). Subjects were also requested to laterally bend the trunk on cue during quiet standing (trunk only, TO). For the TO condition, additional somatosensory cues (see below) were provided to trigger voluntary trunk bending to the ipsilateral (TOI) and contralateral (TOC) directions.

Simultaneous auditory and visual cues were used to trigger trunk bending to the appropriate side at the onset of support-surface movement. The auditory cue consisted of a $50-\mathrm{dB}$ sound pressure level $1000-\mathrm{Hz}$ tone that was produced by either right or left loudspeakers positioned at the same height and $1 \mathrm{~m}$ laterally away from the participants' ears. This auditory cue was automatically switched off when movement sensors (light barriers) detected a lateral movement equivalent to $15^{\circ}$ of trunk flexion. Visual trigger lights were located at eye level, approximately $4 \mathrm{~m}$ in front of the participants. A green light stimulus appeared in the left visual field and at the same time a loudspeaker to the left of the participant sounded when the participant was expected to bend the trunk to the left. (A red light appeared in the right visual field, and the right loudspeaker sounded when bending should be to the right). The instruction to the subject was to flex the trunk to the expected side as rapidly as possible in response to the visual and auditory cues, with
Fig. 1 Stick figures showing movements of a typical subject for all experiment conditions. These are perturbation only (PO figure centre), voluntary trunk bending contralateral (TOC left of centre) and ipsilateral (TOI) to a negligible support-surface tilt, and combined PO and voluntary trunk bending contralateral (CONT leftmost stick figure) and ipsilateral (IPS) to the support-surface tilt. The support-surface tilt was for PO, CONT and IPS conditions in the right-backward LPo direction$113^{\circ}$ (see insert)

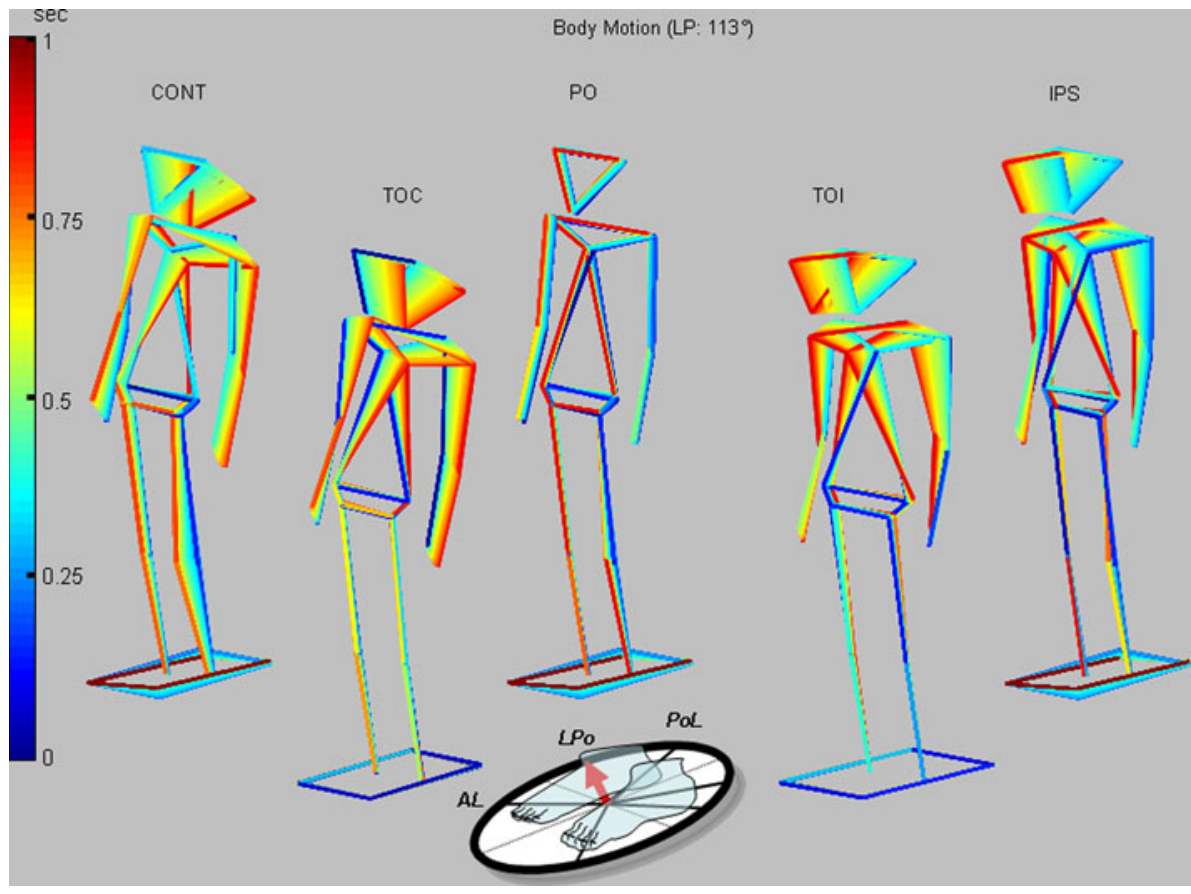


the specific goal to switch off the sound. For consistency, the same auditory cue present in the TO, CONT and IPS conditions sounded at the onset of platform movement in the PO condition as well, but then the specific instruction was to respond naturally to the balance perturbation.

The PO condition was always presented first in order to exclude learning effects of trunk bending conditions on responses. Secondly, 26 TO trials were performed with lateral trunk flexion to $15^{\circ}$. We chose $15^{\circ}$ based on pilot experiments. These showed that $15^{\circ}$ was the maximum lateral flexion subjects were comfortable with. A small (approximately $0.1^{\circ}$ ), just perceptible (see Beule and Allum 2006) pure roll perturbation was presented under TO conditions only, simultaneous with the auditory and visual knee bending prompts in order to have a directional somatosensory cue present without perturbing balance. The 26 TO trials were divided into two sets. Thirteen for bending the trunk ipsilateral (TOI) and 13 trials for bending the trunk contralateral to $0.1^{\circ}$ of tilt (TOC). The order of TOI and TOC series was also randomised across participants. Seven of these small tilt perturbations to the left (TOC) and seven to the right (TOC) followed by 6 to the left (TOI) and 6 to the right (TOI) were presented serially in a randomised order across subjects within each set. Finally, 43 trials under CONT and 43 under IPS conditions were then presented. The order of CONT and IPS presentations was also randomised across subjects. The first trial of each sequence $(158,203,203$ directions for PO, IPS, and CONT conditions, respectively) was analysed separately in order to consider 1st trial effects separate from the main body of data (Keshner et al. 1987; Oude Nijhuis et al. 2009). To minimise fatigue, participants were given a 3-4 min seated rest after each sequence. Perturbations were initiated automatically and were preceded by a random 5-15 s inter-stimulus delay. During this time period, visual feedback of the subjects' own AP and medio-lateral (ML) ankle torque was presented to the subject on a cross with light-emitting diodes. This visual feedback was used to maintain a standardised pre-stimulus subject centre of foot pressure (COP) position across trials. Because the COP was low pass filtered at $5 \mathrm{~Hz}$ (see below), the COP signal provided an approximate measure of centre of mass (COM) position.

\section{Data collection}

Recordings of biomechanical and electromyographic (EMG) data commenced $100 \mathrm{~ms}$ prior to perturbation onset and terminated $1 \mathrm{~s}$ later. To record EMG activity, pairs of silver-silver chloride electrodes were placed bilaterally approximately $3 \mathrm{~cm}$ apart along the muscle bellies of paraspinalis (Para), at the L1-L2 level of the spine, and gluteus medius (Glut Med). Unilateral records on the left side were taken for tibialis anterior (TA), soleus (Sol), biceps femoris (Hamstrings), left rectus femoris (Quadriceps), left medial deltoid (pars acromialis) and external oblique (Ext Obl) muscles. Electrodes were placed along the direction of muscle fibres if muscle bellies were not prominent. EMG recordings were analog band-pass filtered between 60 and $600 \mathrm{~Hz}$, full-wave rectified, and low pass filtered at $100 \mathrm{~Hz}$ prior to sampling at $1 \mathrm{kHz}$.

Full body kinematics was collected using a three-dimensional optical tracking system with 21 infrared-emitting diodes (IREDs) (Optotrak, Northern Digital). The Optotrak cameras sampled the IRED signals at $64 \mathrm{~Hz}$ and were placed approximately 4 metres in front of the subject. IREDs were placed bilaterally on the following anatomical landmarks: frontally at the lateral malleolus; centre of the patella; frontally at the greater trochanter; anterior superior iliac spine; radial styloid process; elbow axis; acromion; chin; angulus sterni; and on a headband placed just above the ears. Three IREDs were placed at the front corners and the left side of the platform to define the pitch and roll movements of the platform. Subjects wore tight-fitting shorts and vests to prevent marker movements with respect to the skin.

Support-surface reaction forces of both feet were measured from strain gauges embedded within the rotating support-surface. The strain gauges were located under the corners of the plate supporting each foot. From forces recorded perpendicular to the support-surface by the strain gauges under the left and right feet and the distances to the centre of ankle joint rotation, the AP and ML ankle torques were calculated for each foot. The torques from the left and right feet were added together, low pass filtered at $5 \mathrm{~Hz}$, and provided as a visual display to the subject as excursions from a centre position, reset as the subjects' comfortable stance position, on two rows of diodes mounted on a cross $4 \mathrm{~m}$ from the subject as described above.

\section{Data analysis}

Trials were excluded when voluntary trunk bending was performed incorrectly. Errors included bending the trunk in the opposite direction to that stipulated by the visual and auditory cues (for example, to the left when cues were presented for the right) or not bending the trunk at all. We used a $15 \%$ trunk velocity threshold (see below) to determine if voluntary trunk movement had occurred and it was in the correct direction. Excluded trials totalled 84 of 4478 trials (1.8\%).

Primary variables of interest were COM, trunk, pelvis, knee joint and upper arm joint, angular velocity, and displacement profiles as well as muscle EMG responses of the legs, arms, and the trunk. Following analog to digital data conversion, kinematic and EMG signals were averaged offline across each perturbation direction. Zero latency was defined as onset platform rotation. Subject average time 
traces were pooled for a single direction for presentation in figures. Responses for directions with the same pitch stimulus component but oppositely directed roll components were pooled for analysis.

\section{Kinematic analysis}

Kinematic data were digitally filtered at $16 \mathrm{~Hz}$ using a zero phase shift, 4 th order, butterworth filter. Total body COM displacement was calculated separately for the AP, ML and vertical directions using a 12 body segment adaptation (see Visser et al. 2008) of a 14 segment model of the human body (Winter et al. 2003). Two trunk segments (upper and lower trunk) were used instead of four. In addition, we calculated the following angular displacements: absolute trunk angle (roll and pitch), absolute pelvis angle (roll and pitch), and ankle, knee, and shoulder joint angles. Absolute rotation angles of the planes defined by trunk and pelvis body segments and the platform surface were defined using 3 or 4 markers on these segments. Arm abduction was calculated as the angle between the upper arm and trunk segment. Knee and ankle joint angles were calculated using angles between segments on either side of the joint (Visser et al. 2008). Stimulus-induced changes were calculated with respect to average values over a pre-trigger time interval of $90 \mathrm{~ms}$ ending $10 \mathrm{~ms}$ prior to stimulus onset. Values of limb, pelvis and trunk kinematics at the time of maximal divergence and at peak velocities were quantified with respect to pre-trigger values and at peak velocities. Peak values were also calculated for the difference in left and right knee flexion and arm abduction.

To determine the onset of trunk motion with respect to the PO condition, a trunk roll velocity threshold of $15^{\circ} / \mathrm{s}$ was used. This threshold was based on the maximum roll velocity for the PO condition and permitted a separation of trunk responses for CONT and IPS conditions with respect to those of PO. Maximum mean roll velocity across directions for PO condition was $9.6 \%$ with a standard error of the mean (SEM) of $0.9^{\circ} / \mathrm{s}$ (therefore, the mean plus 2 SEM equalled $11.4 \%$ s) for all directions.

\section{EMG analysis}

Each EMG response was corrected for background activity by subtracting the average level of pre-stimulus activity measured over a $90-\mathrm{ms}$ period ending $10 \mathrm{~ms}$ prior to perturbation onset. Then, techniques similar to those previously employed (Grin et al. 2007; Küng et al. 2009a, b) were used for analysis. The onset of activity was defined for each muscle based on the perturbation direction showing the greatest amplitude of muscle activity prior to $250-\mathrm{ms}$ poststimulus onset. From the time of peak activity, the analysis algorithm looked backwards in time until activity first reached a value lower than mean pre-stimulus activity plus 2.5 SD. Then, areas were calculated over $130 \mathrm{~ms}$ from this onset time for each individual response. Response areas were also calculated over a fixed interval from 200 to 400ms post-stimulus and as used by Grin et al. (2007) and Küng et al. (2009b), and over a fixed interval from 500 to 800-ms post-stimulus.

\section{Statistics}

First, three-way repeated measures ANOVA's (side: right vs. left $\mathrm{x}$ condition: CONT vs. IPS vs. PO $\times$ direction) were conducted using a significance level of 0.05 in order to justify pooling directions with equal pitch but opposite roll components $\left(45^{\circ}\right.$ and $315^{\circ}, 113^{\circ}$ and $248^{\circ}, 158^{\circ}$ and $203^{\circ}$ ). Having determined that side did not influence the results of our analysis, we concentrated on between-conditions comparisons of PO, CONT and IPS using two preplanned ANOVA's. First, two-way repeated measures ANOVA's (CONT vs. IPS vs. PO $\mathrm{x}$ direction) were conducted on all dependent measures using a significance level of 0.05 . In order to examine how well voluntary and balance correcting responses under CONT and IPS conditions were integrated together by the CNS, we examined with similar two-way ANOVA's the differences between combined and predicted responses, that is IPS vs. PO + TOI and CONT vs. PO + TOC. Significantly different effects between conditions were evaluated within each pooled perturbation direction using Bonferroni post-hoc t-tests.

\section{Results}

Figure 1 shows stick figures of a typical subject for all 5 of the experimental conditions following a tilt in the $113^{\circ}$ (LPo) direction. Our analysis described below addresses three questions. Firstly, whether or not CONT or IPS conditions brought any stability benefits IPS conditions did not. Therefore, we concentrated on the kinematic and muscle responses for the CONT conditions asking whether these were predictable from the TOC and PO conditions (as appears to be the case for the kinematics of Fig. 1); and how well the CONT conditions aid stabilisation of the COM. Finally, we examined how well muscle responses were integrated into one action for CONT conditions.

\section{Kinematics}

\section{Automatic and voluntary trunk lateral flexion responses}

Figure $2 \mathrm{a}$ and $\mathrm{b}$ show trunk roll angle and angular velocity average population traces for a tilt in the $113^{\circ}$ direction. To characterise these traces, average values for all tilt 
(a) Trunk Roll Angle (LP: 113)

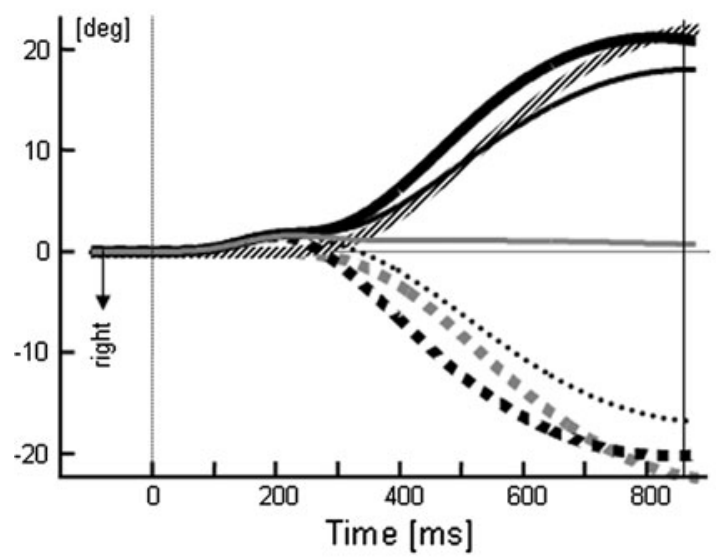

(b) Trunk Roll Angular Velocity (LP: $113^{\circ}$ )

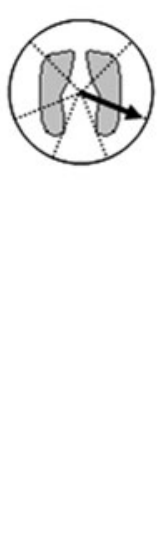

CONT
PO+ TOC
TOC ImImImIn.

PO + TOC

$\triangle \mathrm{CONT}$

$\square$ PO

ZIPS

$\mathrm{PO}+\mathrm{TOI}$

* CONT or IPS $\neq \mathrm{PO} p<0.05$

\# PO + TOC $\neq$ CONT $p<0.05$

$\# \mathrm{PO}+\mathrm{TOI} \neq \mathrm{IPS} \mathrm{p}<0.05$

$\mathrm{TO}=\mathrm{n}=$

(d) Velocity at $\mathbf{4 5 0} \mathrm{ms}$ post stimulus

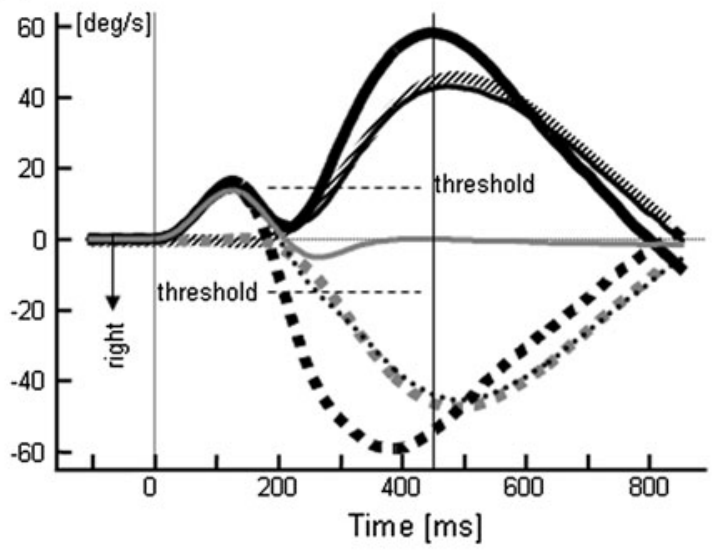

PS $\because \cdots \cdot$

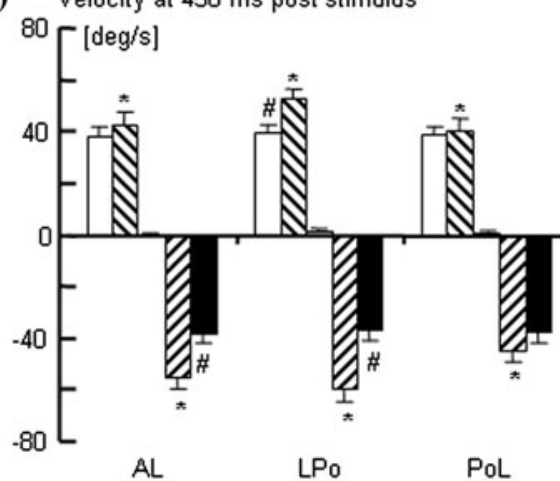

Tilt Direction

Tilt Direction

Fig. 2 Mean population traces of the trunk roll angle (a) and angular velocity (b) across conditions for a backward-right tilt. The short dotted horizontal lines in (b) mark the threshold used as onset of the voluntary trunk bending exceeding that of the PO condition $15 \%$. Column heights represent the population mean values under each condition (bars over the columns, standard errors of the means (SEM))

directions were computed at 850 and $450 \mathrm{~ms}$, as shown, respectively, in Fig. 2c and d. As indicated in Fig. 2, trunk tilt was opposite to that of the stimulus for the PO condition, but much smaller compared to the trunk bending conditions. Initially, trunk roll was approximately $2^{\circ}$ for PO conditions but reduced to less than $1^{\circ}$ by $850 \mathrm{~ms}$ depending on the perturbation direction (AL: $0.23 \pm 0.20^{\circ}$; LPo: $0.81 \pm 0.23^{\circ}$; PoL: $\left.0.15 \pm 0.13^{\circ}\right)$. Consistent with previous studies (Allum et al. 2008; Carpenter et al. 2001), this resulted in a direction effect for trunk roll angle $(F(2,47)=12.9, P<0.001)$, with LPo directions inducing the greatest trunk roll. For TO, trunk roll angles of $16.9^{\circ}\left( \pm 0.7^{\circ}\right)$ were acquired. Fifteen degrees of trunk roll flexion was requested from the subjects. When voluntary trunk roll flexion occurred with support-surface tilt (CONT for roll angle (c) and angular velocity (d). Asterisks (*) on the columns indicate significant differences between the PO and CONT or PO and IPS conditions, a gate (\#) symbol indicates significant differences between the CONT and (PO + TOC) or IPS and (PO + TOI) predicted mean values

and IPS conditions), greater trunk movements were observed than for TO across all directions (condition effect: CONT vs TOC, $F(1,88)=4.6, P=0.045$; IPS vs. TOI, $F(1,91)=4.08, P=0.05)$. Under CONT, trunk roll angle at $850 \mathrm{~ms}$ equalled for $\mathrm{AL} 19.5 \pm 0.9^{\circ}$, for LPo $20.8 \pm 0.9^{\circ}$, and for PoL $16.3 \pm 1.1^{\circ}$. For IPS, the roll angles at $850 \mathrm{~ms}$ were for $\mathrm{AL}-20.6 \pm 1.0^{\circ}$, for $\mathrm{LPo}-20.1 \pm 0.9^{\circ}$, and for $\mathrm{PoL}-17.0 \pm 0.7^{\circ}$ (in the opposite direction to CONT). In all directions, except PoL, trunk roll angles were significantly different from those of TOC for both CONT and TOI for IPS. A directional effect of trunk roll was recorded for $\operatorname{CONT}(F(2,43)=17.7, P<0.001)$ and $\operatorname{IPS}(F(2,46)=22.1$, $P<0.001)$. Amplitudes of trunk roll angle for CONT and IPS, were significantly greater than predicted values, that is, $\mathrm{PO}+\mathrm{TOC}$ or $\mathrm{PO}+\mathrm{TOI}$, respectively, $(F(1,89$ to 92$) \geq 5.2$, 
$P \leq 0.03)$ with the exception of backward perturbation directions, PoL (see Fig. 2c).

Figure $2 \mathrm{~b}$ shows average population angular velocity traces of trunk roll. In the CONT and IPS conditions, an early phase of trunk roll velocity opposite to tilt during the first $200 \mathrm{~ms}$ precedes a larger phase of trunk roll velocity. The early phase was also observed for PO but not for TO conditions. When the time to reach over $\pm 15 \%$ s-threshold for voluntary action (marked in Fig. $2 b$ by horizontal lines) was considered, times for IPS occurred significantly earlier than for CONT $(F(1,88)=8.1, P=0.006)$. The times for IPS were AL $206 \pm 1.1 \mathrm{~ms}$, LPo $194 \pm 1.0 \mathrm{~ms}$, and PoL $240 \pm 1.2 \mathrm{~ms}$. These times were earlier than for CONT by $70.1 \pm 12.7 \mathrm{~ms}$, for AL by $29.8 \pm 15.1 \mathrm{~ms}$ for LPo, and by $47.2 \pm 16.0 \mathrm{~ms}$ for PoL. The onset times for the CONT condition were equal to those of TOC except for the LPo direction for which the onset was later by $46.9 \pm 13.5 \mathrm{~ms}$, $P=0.007$. Lateral trunk flexion for the IPS condition reached the $-15 \%$ threshold faster than under TOI condition $(F(1,91)=17.315, P<0.001)$ : AL by $67.3 \pm 10.6 \mathrm{~ms}$, LPo by $79.5 \pm 10.9 \mathrm{~ms}$, and PoL by $35.4 \pm 9.4 \mathrm{~ms}$ ), possibly because the IPS velocity profile coincided with a negative pulse of trunk velocity present under the PO condition (see Fig. 2b).

Trunk velocity reached a peak around $420 \mathrm{~ms}$ on average for the IPS condition (AL $419 \pm 2.0 \mathrm{~ms}$, LPo $404 \pm 1.9 \mathrm{~ms}$. PoL $432 \pm 2.0 \mathrm{~ms}$ ) slightly faster than the peak for the CONT condition (see Fig. 2b). However, when considering the times between $\pm 15 \%$ s thresholds and peak velocity, no significant difference could be found between IPS and CONT conditions. Furthermore, peak amplitude did not differ between CONT and IPS conditions in any direction, reaching $56.1 \pm 3.1 \%$. However, velocity amplitudes at $450 \mathrm{~ms}$ were larger than predicted for AL and LPo directions (see Fig. 2d). Thus, generally, voluntary trunk flexion under CONT and IPS conditions was larger than predicted in the AL and LPo directions, and occurred earlier when executed in the destabilising (IPS) direction.

\section{COM displacements}

Figure 3 shows population position traces for $\mathrm{COM}$ in the ML (A) and in the AP (B) directions following a lateral tilt $\left(113^{\circ}\right)$. The ML displacement of the COM (measured at 850-900 ms) showed a medial directed benefit to balance recovery under the CONT condition by some $37 \mathrm{~mm}$ - see Fig. $4 \mathrm{a}$ and c. Under the IPS condition, a marked disadvantage was seen (by $30.5 \pm 1.4 \mathrm{~mm} ; F(2,132)=298.8$, $P<0.001$-Figs. 3a and 4a). Because of this clear instability compared to the PO condition, we focussed most of our analysis of COM velocity and body segment movements on the CONT condition. The gain in COM stability obtained with voluntary trunk lateral flexion under the CONT condition is compared with that of unilateral arm bending (Grin et al. 2007) and knee bending (Küng et al. 2009b) in Fig. 4c.

\section{COM position and velocity}

ML COM position under the CONT condition clearly benefited from trunk bending uphill with an uphill shift (AL: $39.7 \pm 2.6 \mathrm{~mm}$, LPo: $35.5 \pm 2.6 \mathrm{~mm}$, PoL: $35.7 \pm 2.3 \mathrm{~mm}-$ see Fig. 4a) that was as predicted for CONT conditions (Fig. 4a). For the very first trial, a PoL perturbation, the shift was not as large (Fig. 4b). The improvement in ML $\mathrm{COM}$ position for CONT was significantly greater than that
Fig. 3 Mean population traces of ML (a) and AP (b) COM position for the different experimental (PO, CONT, IPS) and predicted $(\mathrm{PO}+\mathrm{TOC}$, $\mathrm{PO}+\mathrm{TOI})$ conditions in response to a backward right $\left(113^{\circ}\right.$ direction) perturbation

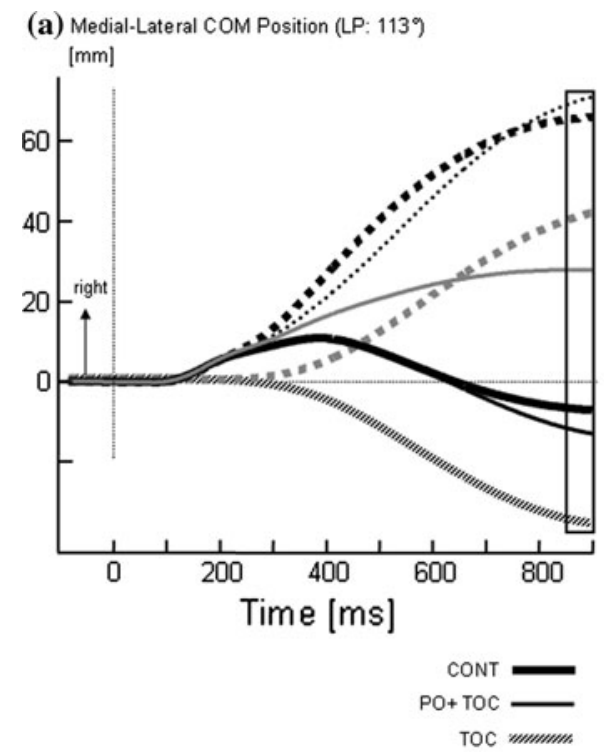

(b) Anterior-Posterior COM Position (LP: 1139)

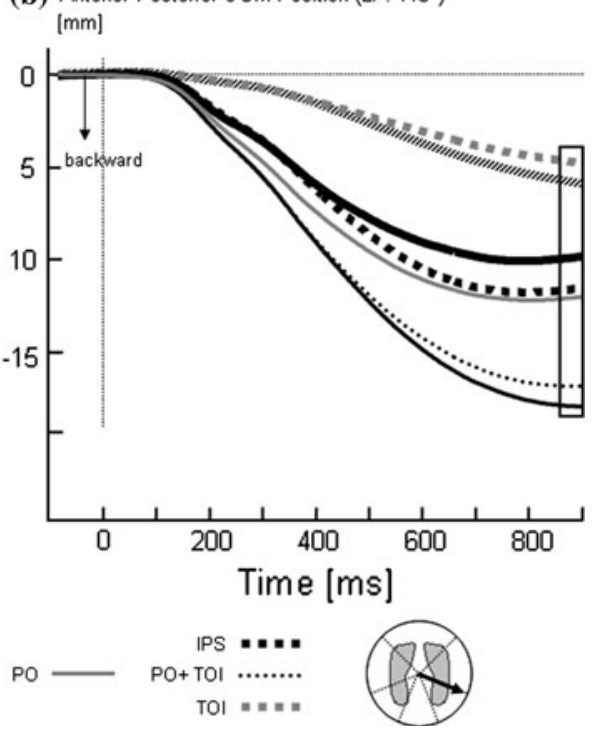


(a) COM ML Displacement at $850 \mathrm{~ms}$

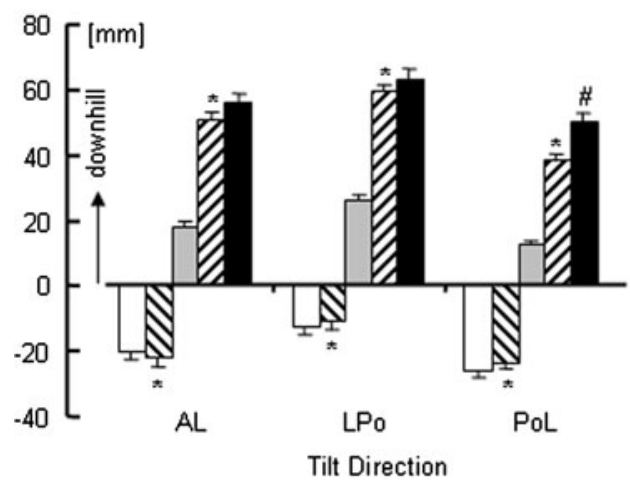

$\begin{array}{ll}\text { * CONT or IPS } \neq \text { PO } p<0.05 & \square \text { PO + TOC } \\ \text { \# PO + TOI \# IPS } p<0.05 & \square \text { CONT } \\ \text { (d) COM AP Displacement at } 850 \mathrm{~ms} & \square \text { PO + TOI }\end{array}$

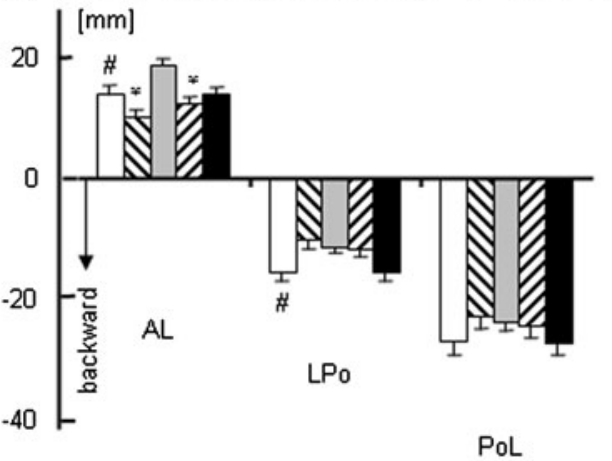

Tilt Direction (b)

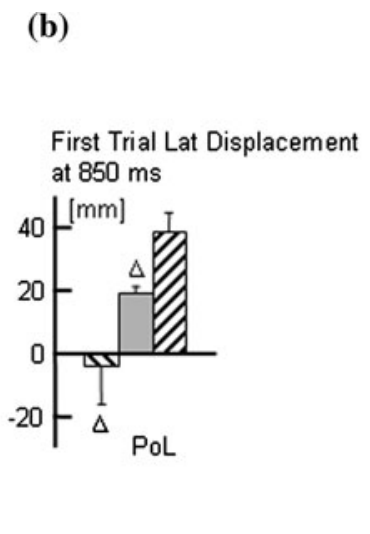

$\Delta$ First $\neq$ Random $p<0.05$

(e) First Trial AP Displacement at $850 \mathrm{~ms}$

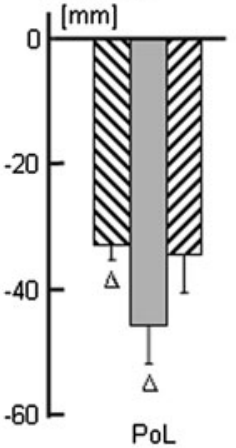

(c) Reduction in ML COM downhill Displacement PO - UK/PO - UA / PO - UT

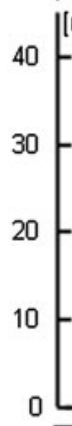

$[\mathrm{mm}]^{*}$

臣 Uphill Unilateral Knee Bending: UK

Uphill Unilateral Arm Abduction: UA

W Uphill Trunk Bending: T

* $T \neq U A$ and $U K p<0.05$

\# $\mathrm{T} \neq$ UK $p<0.05 \quad \triangle$ UK $\neq$ UA $p<0.05$

(f) Difference in AP COM Displacement for CONT $\mathrm{PO}$ - UK/PO - UA/PO - T

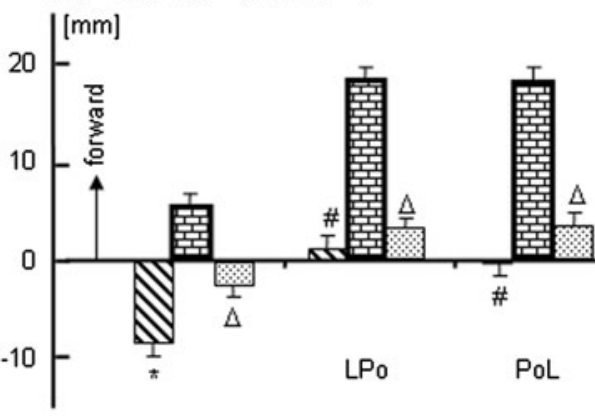

AL

Tilt Direction
Fig. 4 Mean (and SEM) population values over 850-900 ms for COM displacements under different conditions (PO, CONT, IPS) as well as predicted values $(\mathrm{PO}+\mathrm{TOC})$ and $(\mathrm{PO}+\mathrm{TOI})$. The upper rows of column plots $(\mathbf{a}, \mathbf{b}, \mathbf{c})$ provide $\mathrm{ML}$ values, the lower row $(\mathbf{d}, \mathbf{e}, \mathbf{f}) \mathrm{AP}$ values. $\mathbf{b}$ and $\mathbf{c}$ show 1 st trial effects for the PoL direction. $\mathbf{c}$ and $\mathbf{f}$

achieved with unilateral arm raising (Grin et al. 2007) or unilateral knee bending (Küng et al. 2009b) under the CONT condition-see Fig. 4c.

Figure 5a displays the COM ML velocity profiles for the right LPo direction. At the time of the first peak in COM ML velocity around 170-ms post-stimulus, no condition effect on the responses was noted. This first peak was followed in PO conditions by a second peak at around 320-ms post-stimulus but a decrease in COM ML velocity under CONT conditions (see vertical line at $320 \mathrm{~ms}$ in Fig. 5a). At this time point, significant differences in $\mathrm{COM}$ velocities became evident across trunk bending conditions. A threeway ANOVA revealed a main condition effect between PO, CONT and IPS $(F(2,132)=82.3, P<0.001)$. COM velocity for the IPS condition involved a greater tilt velocity downhill than for the PO condition and therefore more unstable (mean difference: $43.3 \pm 2.9 \mathrm{~mm} / \mathrm{s}$ ). The COM compares the changes in ML and AP displacements with respect to $\mathrm{PO}$ conditions under CONT (uphill) conditions for voluntary trunk (T), unilateral knee (UK), and unilateral arm abduction (UA). The UK data is from Küng et al. 2009b, UA data from Grin et al. 2007). For other details, refer to the legend of Fig. 2

velocity was smaller and therefore more stable than $\mathrm{PO}$ for the CONT condition, (mean difference: $22.6 \pm 2.5 \mathrm{~mm} / \mathrm{s}$ see Fig. 5b). The values for the CONT condition were as predicted from the $\mathrm{PO}+\mathrm{TOC}$ conditions (see Fig. 5a). The effect on COM velocity at $320 \mathrm{~ms}$ for the CONT condition was similar to that obtained with unilateral knee bending or arm raising to the CONT side (Grin et al. 2007; Küng et al. 2009b), except for the PoL direction for which unilateral trunk bending was slightly better (Fig. 5b).

In contrast to voluntary arm raising and knee bending, a further major improvement in COM velocity occurred with CONT trunk bending. This was characterised by a peak in COM velocity in a direction opposite to that of the perturbation at $540 \mathrm{~ms}$ (see Fig. 5a), that is uphill. Measurement at this time showed a condition effect between the PO, CONT and IPS conditions $(F(2.132)=268.855$, $P<0.001$-see Fig. 5c). Furthermore, in contrast to the 
(a) Medial-Lateral COM Velocity (LP: $113^{\circ}$ )
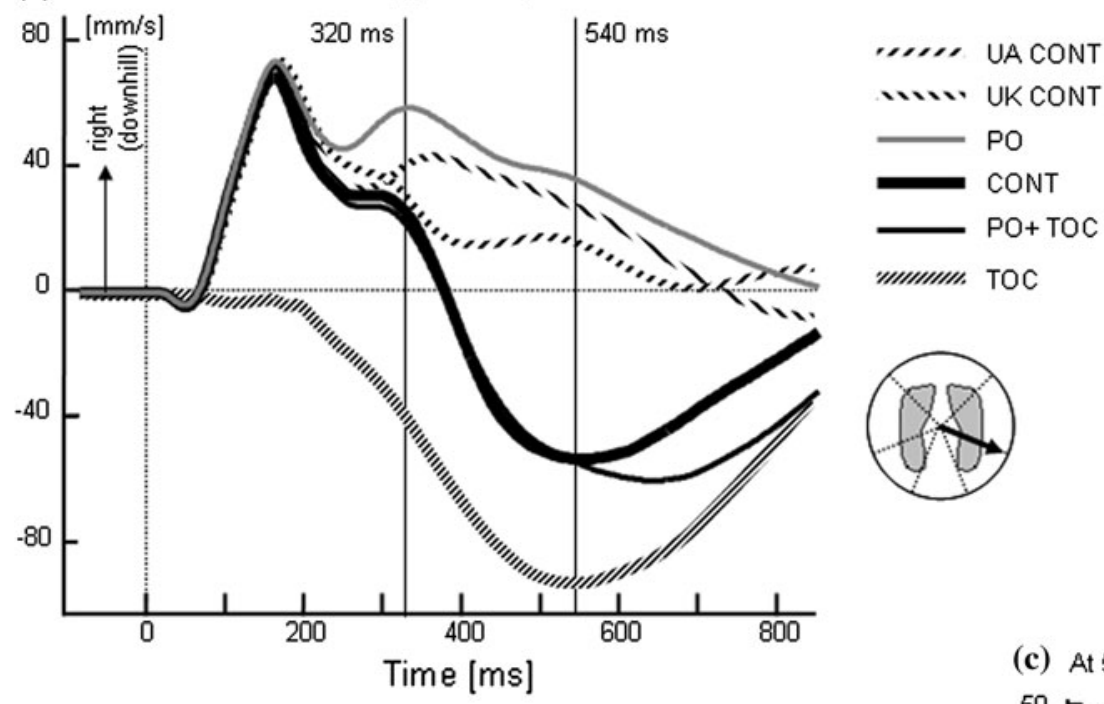

(b) At $320 \mathrm{~ms}$ post stimulus

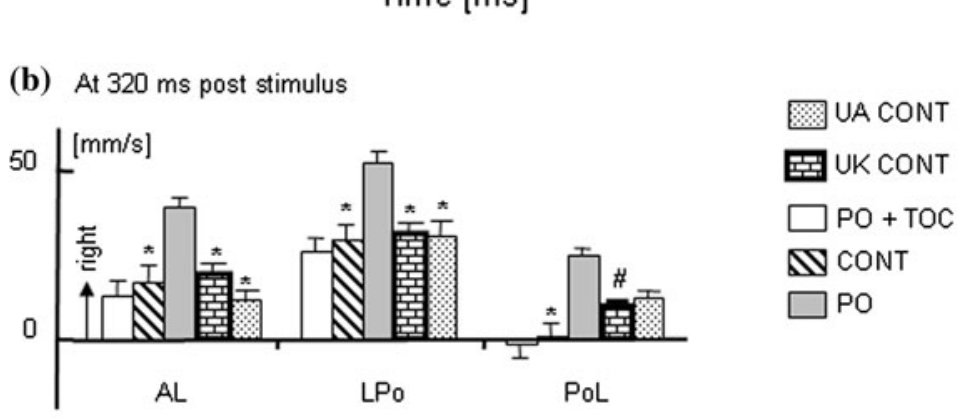

Tilt Direction (c) At $540 \mathrm{~ms}$ post stimulus

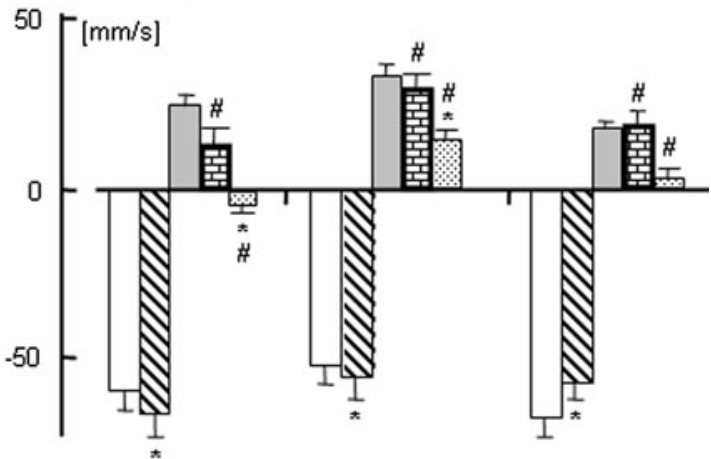

AL

LPo

Tilt Direction
Fig. 5 Mean population traces of ML COM velocity across conditions for a backward-right tilt (a) and mean population values of ML COM velocity across directions and conditions measured at $320 \mathrm{~ms}$ (b) and

values at $320 \mathrm{~ms}$, the improved stability of COM ML velocity was much greater than that achieved with unilateral arm or knee bending (Fig. 5c). However, values were as predicted from $\mathrm{PO}+\mathrm{TOC}$ conditions. Thus, in general, voluntarily bending the trunk uphill at onset of the balance perturbation had a very positive effect on ML COM motion. Thus, it is not surprising that a high correlation between trunk roll velocity at 450 -ms post-stimulus compared to COM velocity at $540 \mathrm{~ms}$ was established under CONT conditions $(R=0.98, P=0.001)$, whereas under PO conditions no significant correlation existed.

\section{AP COM position}

Voluntary trunk flexion brought no significant improvement in AP stability. In the AL direction under CONT conditions, the AP COM position was shifted backwards by $8.4 \pm 1.3 \mathrm{~mm}$ compared to PO. No significant differences occurred for backward directed tilts LPo and PoL (Fig. 4d).
$540 \mathrm{~ms}$ (c) as marked by the vertical lines in (a). For details, refer to the legends of Figs. 2 and 4

For the very first trial, slightly less instability occurred under CONT conditions in comparison to the 1 st $\mathrm{PoL}$ directed PO trial, but the instability was nonetheless more than for the rest of the CONT trials (Fig. 4e). The lack of improvement in backward AP stability under CONT trunk bending was significantly different from that occurring with unilateral knee bending (Fig. 4f). As Fig. 4f shows the action of voluntary knee bending produced an improvement in AP stability in the form of a forward shift of the COM for all perturbation directions.

\section{Pelvis displacements}

We examined pelvis angular and linear displacements because of the proximity of the COM to the pelvis. The pelvis initially rolled and was shifted in the same lateral direction as the support-surface rotation (Fig. 6a and c). The CONT condition created a shift in pelvis angle back towards the pre-stimulus position as measured at $750 \mathrm{~ms}$ 
(a) Roll Angle (LP: $113^{\circ}$ )

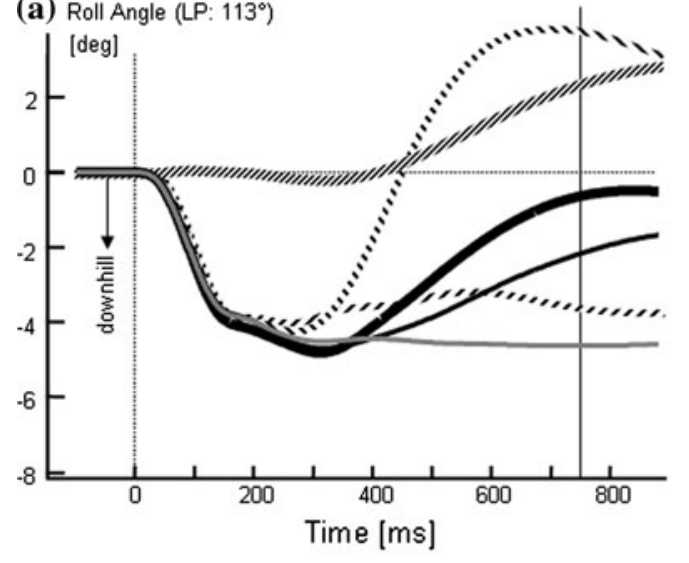

(c) Pelvis Medial-Lateral horizontal Velocity (LP: $113^{\circ}$ )

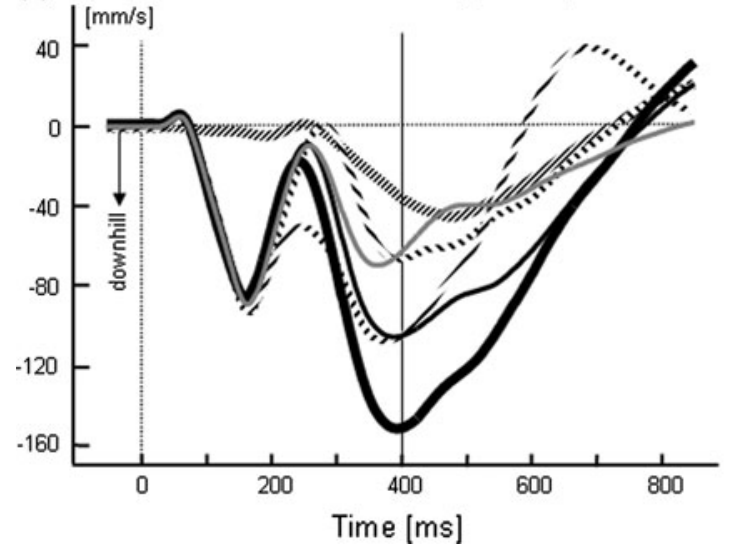

Fig. 6 Mean population traces of pelvis roll angle and pelvis lateral velocity (a and $\mathbf{c}$, respectively). Mean values of pelvis roll are shown in (b) across directions and conditions and measured at $750 \mathrm{~ms}$ (see vertical line in a) when pelvis roll displacement plateaued. In (d), mean values of ML pelvis lateral velocity at $400 \mathrm{~ms}$ across conditions are

(see vertical line in Fig. 6a). The CONT condition reduced the initial pelvis downhill roll more than predicted regardless of the tilt direction (Fig. 6b). Examination of pelvis roll angular velocity at 450 -ms post-stimulus reflected this finding, with the uphill velocity being greater than predicted by the PO + TOC conditions (data not shown). Interestingly, the shift in pelvis roll to the uphill side under the CONT condition was less than with unilateral knee bending (see UK values in Fig. 6b) but greater than for unilateral arm raising (UA values in Fig. 6b).

For the CONT, PO, TOC conditions, the pelvis moved horizontally to the downhill side. This lateral shift of the pelvis, downhill, was increased in CONT as predicted, except for LPo condition where the pelvis was shifted more than predicted by $8.9 \pm 2.2 \mathrm{~mm}$ (as can be assumed from the lateral velocity traces in Fig. 6c and values in Fig. 6d). ML horizontal velocity of the pelvis peaked at approximately 400-ms post-stimulus (see vertical line in Fig. 6c). The amplitude of this peak was increased for the CONT (b) Pelvis Roll at $750 \mathrm{~ms}$ post stimulus
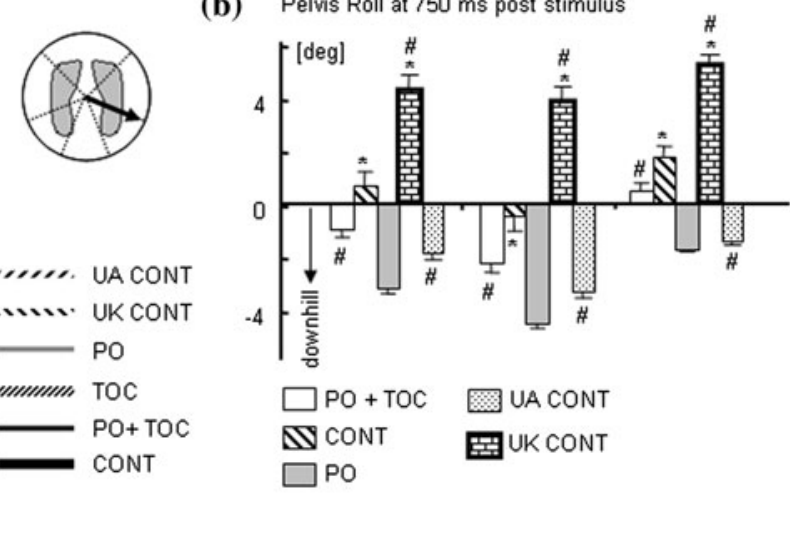

(d) Pelvis Medial-lateral Displacement Velocity

at $400 \mathrm{~ms}$ post stimulus

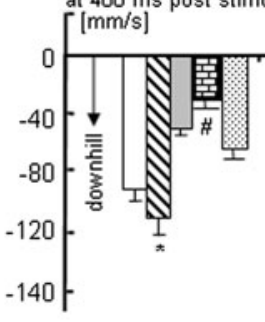

$\mathrm{AL}$

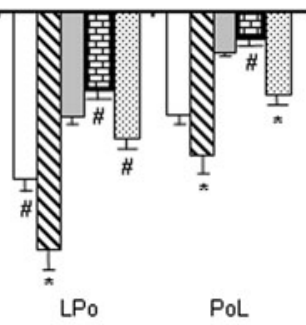

Tilt Direction

$$
\begin{aligned}
& * \mathrm{PO} \neq \text { CONT } p<0.05 \\
& \# \text { CONT } \neq \mathrm{PO}+\text { TOC or UA CONT or UK CONT } p<0.05
\end{aligned}
$$

shown. At $400 \mathrm{~ms}$, the lateral velocity peaked (see vertical line in c). Data has been added for comparison of pelvis movements for voluntary unilateral knee bending (UK) and arm abduction (UA). For details, refer to legends of Figs. 2 and 4

condition by $57.0 \pm 6.5 \mathrm{~mm} / \mathrm{s}$ compared to the $\mathrm{PO}$ condition $(F(2,141)=72.010, P<0.001)$. The PO + TO conditions predicted smaller changes in peak amplitudes, than occurred for the CONT condition, by $26.6 \pm 4.1 \mathrm{~mm} / \mathrm{s}$, on average. In contrast to voluntary unilateral knee bending which produced a reduction in pelvis lateral velocity (Fig. 6d), voluntary trunk bending caused an increase in this measure and for this reason cannot underlie stabilisation of the COM.

\section{Knee flexion}

As voluntary unilateral knee bending provides a stabilising shift in AP COM displacement under CONT conditions (see Fig. 4f), we also examined the knee bending present with voluntary shifts of the trunk uphill. Specifically, we investigated the difference in left and right knee flexion to determine if stabilising effects of knee flexion on COM shifts were present (Küng et al. 2009a). The differences of 
left and right knee flexion showed greater stabilising knee motion for the CONT trunk bending condition than for the PO condition. The greatest knee flexion angle difference for the CONT condition was seen at approximately 700-ms post-stimulus (data not shown). At this time point, knee flexion difference for the CONT condition was greater than for the PO condition by, on average, $6.4 \pm 0.6^{\circ}$; $F(2,140)=19.5, P<0.001)$, and greater than predicted from the PO + TOC conditions by $5.5 \pm 0.6^{\circ}(P<0.001)$.

Under the CONT condition, knee flexion was more stabilising than for PO, because the knee flexion velocity difference between the left and right knees was increased; however, not as much as for voluntary knee flexion (Küng et al. 2009b, see Fig. 7a). The knee velocity difference profiles consist of two peaks at 250 and $430 \mathrm{~ms}$ for voluntary trunk bending but one larger peak for voluntary knee bending (see vertical lines in Fig. 7a). At $250 \mathrm{~ms}$, there were no improvements in knee velocity compared to the PO condition (see Fig. 7b). At 430-ms post-stimulus, a more stabilising knee flexion velocity difference was detected for the trunk bending CONT condition compared to the PO condition by, on average, $17.8 \pm 2.4 \%$ - see Fig. 7 c. Because little knee flexion velocity occurred at $430 \mathrm{~ms}$ under the TOC condition (see Fig. 7a), predicted knee velocity differences from the $\mathrm{PO}+\mathrm{TOC}$ conditions were similar to $\mathrm{PO}$ and therefore CONT values were significantly greater than for the PO condition by $15.4 \pm 2.3 \% \mathrm{~s}(F(1,93)=12.8$, $P=0.001$-see Fig. 7c). Knee motion at 430 -ms post-stimulus for CONT correlated well with COM ML velocity at 540 -ms post-stimulus $(R=0.995, P<0.001)$.
Muscle responses

We analysed muscle activity over 3 intervals (see Figs. 8 and 9), an early balance correcting activity, (see Fig. 8a), a second interval of activity from 200 to $400 \mathrm{~ms}$ associated with voluntary trunk bending but not seen under PO conditions and a later interval of 500-800 ms associated with stabilising activity. Generally under the CONT condition, muscle activity was as predicted from the PO and TOC conditions.

\section{Trunk muscles}

Figures 8 and 9 illustrate the left Para and left Ext Obl muscle responses for the two ML directions $\left(113^{\circ}\right.$, left and $248^{\circ}$, right). In the first interval of $130 \mathrm{~ms}$ (81$211 \mathrm{~ms}$ for Para, and 43-173 ms for Ext Obl) covering the early pulse of muscle balance correcting activity, differences between CONT and PO conditions, and between CONT and predicted (PO + TOC) values, were noted (see Figs. 8 and $9 \mathrm{a}$ and b). However, these differences were not significant.

A different pattern emerged for the interval $200-400 \mathrm{~ms}$ post-stimulus. Significant response differences were observed for uphill muscles between CONT and PO conditions (see Figs. 8c and 9c). That is, tilt of the platform to the right caused larger responses in the left (uphill) trunk muscles (see Figs. 8a and 9a). These CONT responses were larger than predicted $(\mathrm{PO}+\mathrm{TOC})$ but not significantly different (see Figs. 7c and 9c).
Fig. 7 Mean population traces of the difference of the left and right knee flexion velocity across conditions (a) and mean values across directions and conditions measured at $250 \mathrm{~ms}$ (b) and $430 \mathrm{~ms}$ (c). These times are marked by vertical lines in (a). For details, refer to legends of Figs. 2, 4 and 6

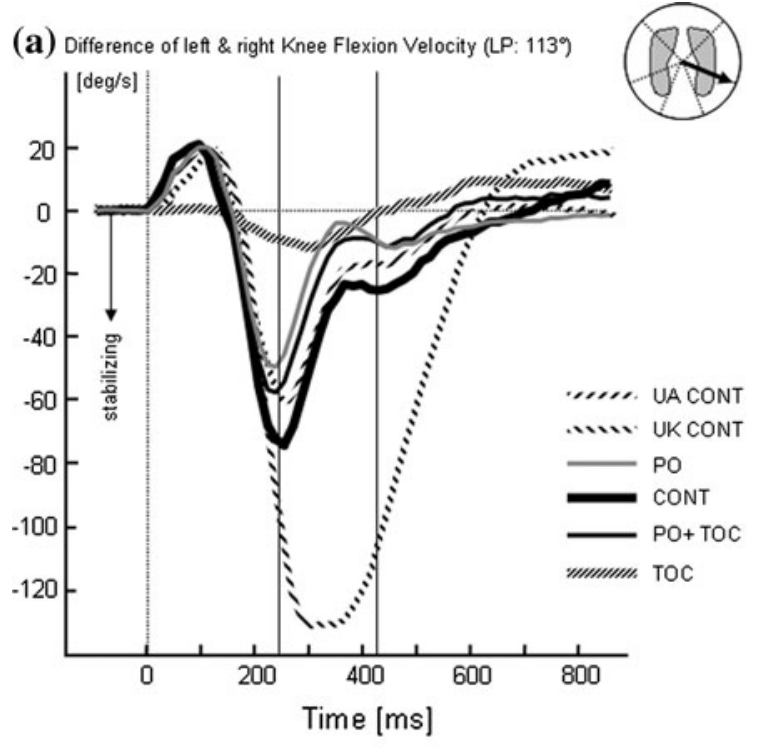

(b) At $250 \mathrm{~ms}$ post stimulus

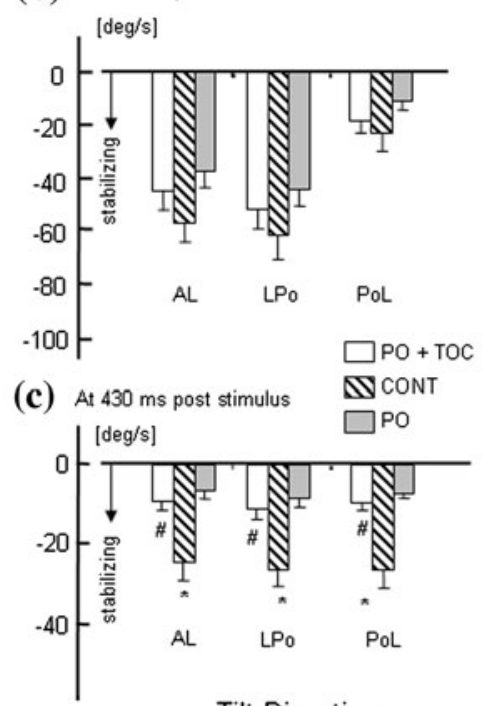

Tilt Direction

* PO $\neq$ CONT or UA CONT or UK CONT $p<0.05$

\# CONT $\approx$ PO+TOC $p<0.05$ 

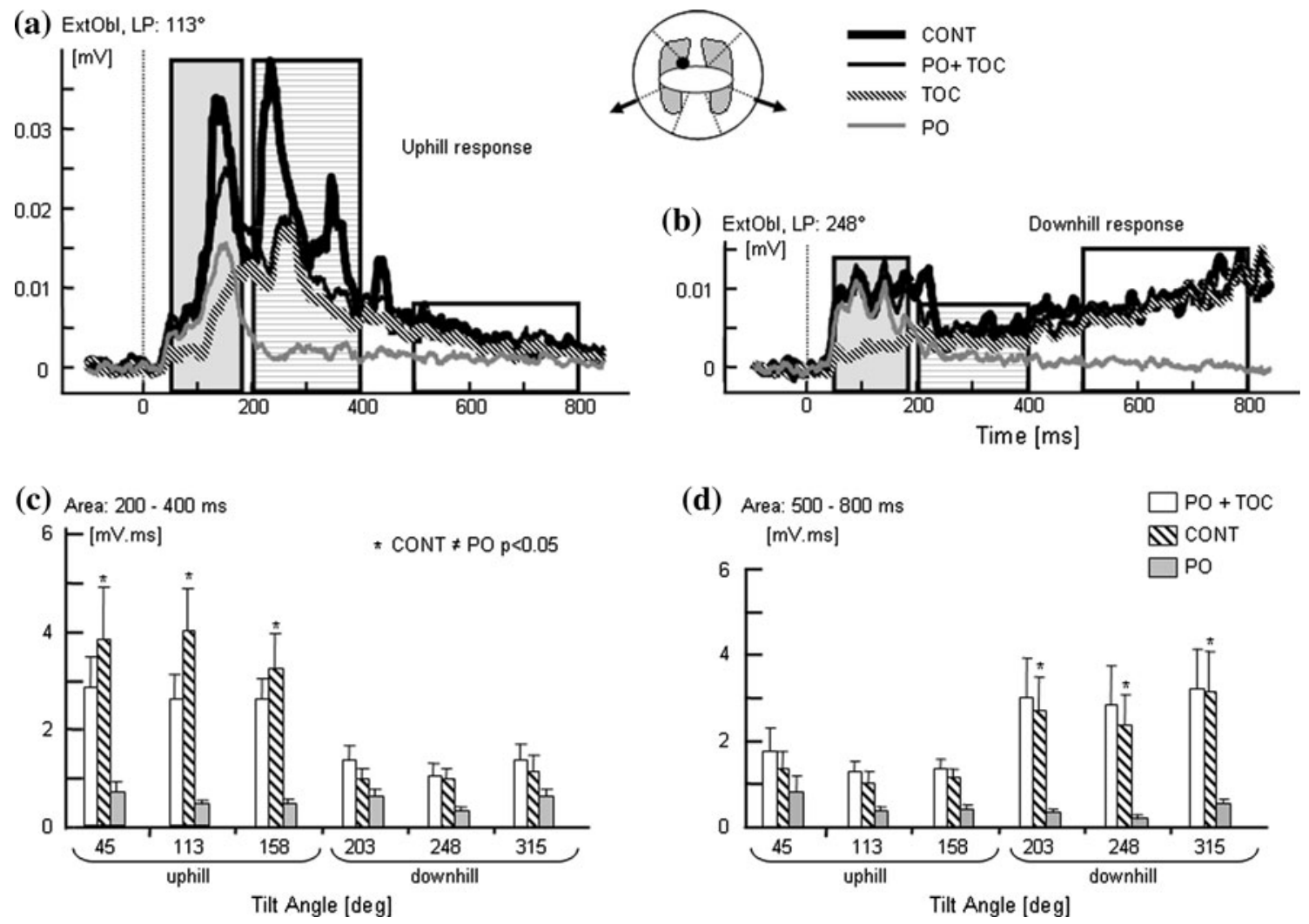

Fig. 8 Changes in left external oblique activity under CONT trunk bending conditions compared to PO, TOC and predicted values $(\mathrm{PO}+\mathrm{TOC})$. The upper set of mean population traces shows the effect of EMG responses from the left external oblique for a backward-right (a) and backward-left (b) tilt when the muscle is the uphill and down-

hill muscle, respectively. Population means and SEM of two different integration intervals across directions and conditions are shown by column plots. The response directions have been labelled uphill or downhill as appropriate

Over the later stabilising interval $(500-800 \mathrm{~ms}$ poststimulus), trunk muscles showed increased activity under CONT compared to $\mathrm{PO}$ conditions when these were the downhill muscles (see Figs. 8d and 9d), presumably to resist the tilt of the trunk uphill. The increase in activity under CONT conditions was as predicted (Figs. 8d and 9d). Activity over this same period in the uphill muscle did not differ between CONT and PO conditions. Therefore, activity in the 500-800-ms period was generally less than predicted, but only significantly less in the paraspinal muscles (see Fig. 9d).

Trunk muscles under voluntary trunk bending (TO) conditions had a similar burst of anticipatory postural activity as seen with voluntary knee bending (Küng et al. 2009b). The onset times of trunk muscle activity for the PO and CONT conditions were $94.6 \pm 3.7$ and $101.0 \pm$ $4.0 \mathrm{~ms}$, respectively, for paraspinals, and $64.3 \pm 4.5$ and $63.8 \pm 4.8 \mathrm{~ms}$, respectively for external oblique. These onset times of muscle activation were slightly earlier than for TO conditions; in paraspinals, by $20.8 \pm 0.6 \mathrm{~ms}$ $(P<0.001)$, and in external oblique, by $24.3 \pm 0.7 \mathrm{~ms}$ $(P<0.001)$.

\section{Leg and arm muscles}

For the upper leg muscles Quad and Ham and upper arm muscles (Deltoid), we found no differences between CONT and predicted $(\mathrm{PO}+\mathrm{TOC})$ conditions. Muscle responses for the CONT conditions showed a trend to be greater than those of PO conditions but due to response variability this difference did not reach significance.

\section{Discussion}

Our results indicate that cued voluntary trunk bending executed in the uphill direction at onset of a tilt perturbation of the support-surface is well integrated into ensuing balance corrections. The stabilising effect on the COM consists of two phases. An early phase peaking in COM velocity around $320 \mathrm{~ms}$ was very similar to that of voluntary unilateral knee bending and arm abduction (Küng et al. 2009b; Grin et al. 2007). A second phase in COM velocity peaking around $540 \mathrm{~ms}$ was unique to lateral trunk bending and brought a greater amount of stability. Our key finding was 

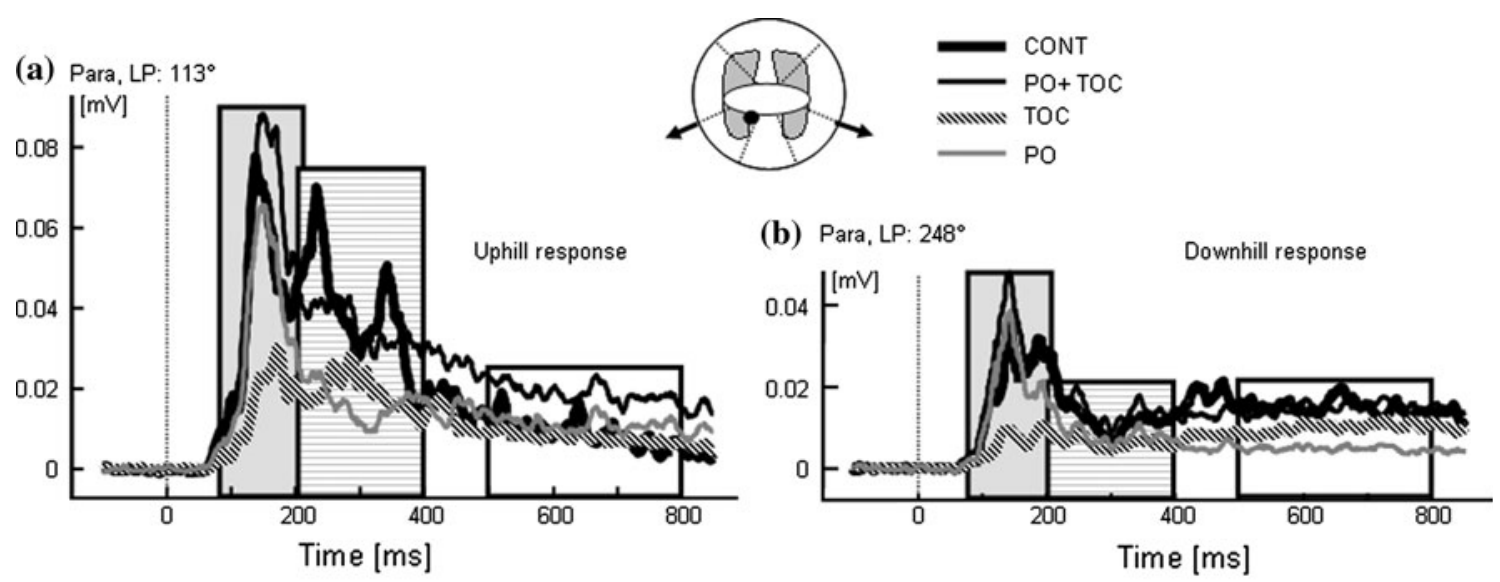

(c) Area: $200-400 \mathrm{~ms}$

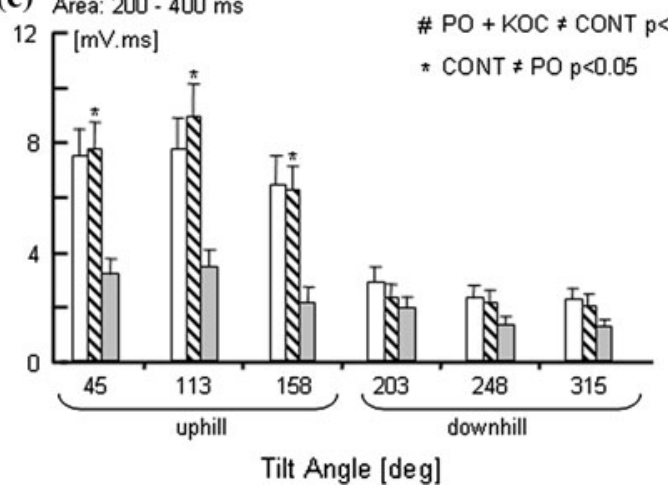

(d) Area: $500 \cdot 800 \mathrm{~ms}$

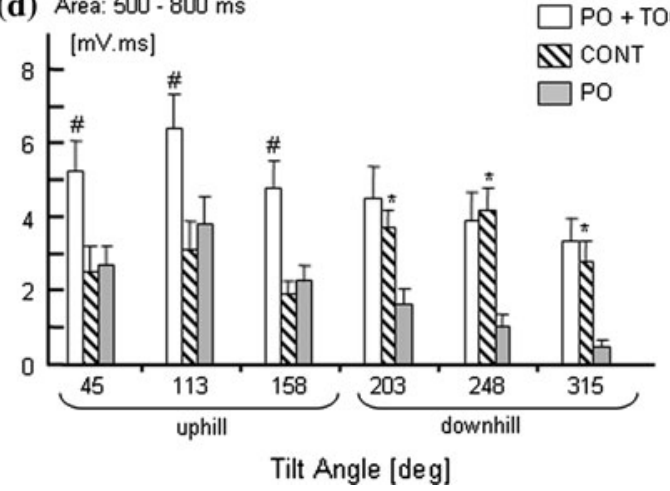

Fig. 9 Changes in left Paraspinal muscle activity with trunk bending conditions. The layout of the figure is identical to that of Fig. 9

that trunk bending and in parallel knee action was correlated with improvements at these time points. In total, the changes in medially directed improvements with trunk bending were greater than those of uphill knee bending. Changes in COM velocity at the 320 and 540-ms time points were reversed into destabilisation when bending the trunk downhill. This reversed action is in contrast to that of unilateral knee bending for which both bending the uphill and downhill knee brought improvements in stability, albeit greater for the uphill knee (Küng et al. 2009b). Bending the uphill knee also brought improvements in AP COM stability not seen with lateral bending of the trunk uphill. The present results have important implications for rehabilitation strategies which could be pursued with those prone to fall, and provide insights into the action of the CNS when executing motor programs based on feedback and feedforward concepts.

When executing arm and leg movements during quiet stance and as parts of balance corrections in response to support-surface movements, a reduction in COM displacement is achieved primarily through active control of trunk orientation with respect to the direction of COM displacement (Wu 1998; Pozzo et al. 2001; Hughey and Fung 2005; Preuss and Fung 2008; Küng et al. 2009a). Specifically for balance corrections, the CNS actively controls the direction of trunk flexion to be opposite to that of the perturbation direction (Carpenter et al. 1999) but with a different timing for roll and pitch directions. This direction dependence is primarily biomechanically determined. The flexibility of the trunk on the pelvis is greater and occurs earlier in the roll other than the pitch direction (Carpenter et al. 1999; Allum et al. 2003, 2008). The strategy used to respond to lateral tilt is to maintain trunk tilt, induced biomechanically by the perturbation, in the uphill direction (Allum et al. 2003). This is an adequate stable strategy in the young, but inadequate in the elderly, particularly if they have pathologies that increase trunk stiffness with respect to movement on the pelvis because then the trunk hardly tilts uphill (Allum et al. 2002; Carpenter et al. 2004). The result in the elderly and patients is a trunk displacement in the direction of an impending fall (Allum et al. 2002). A countervening strategy in this case would be a supplementary voluntary flexion uphill. Although the current study was performed on young participants, we believe that the elderly could perform cued voluntary flexion of the trunk and thereby improve their lateral stability too.

With this and previous research (Grin et al. 2007; Küng et al. 2009b), we have shown that providing cues at the 
onset of a balance disturbance can improve balance control in young subjects. The cues provided were specific to the direction of imbalance. While it remains to show that similar mechanisms would be effective in the elderly, current research on providing cues during stance and gait tasks to improve balance control (Davis et al. 2010; Verhoeff et al. 2009) suggests that this is the case.

The stabilising action of the knees and arms on the COM described here following voluntary lateral trunk bending uphill was similar to that achieved with voluntary unilateral knee bending and arm abduction, however with reduced amplitudes (Küng et al. 2009b; Grin et al. 2007). Although a slight increase stabilising knee flexion in comparison with the perturbation only condition was observed at $250 \mathrm{~ms}$, it is probable, given the correlations we observed between knee flexion and COM velocity, that this knee flexion combined with early trunk roll was responsible for the first phase in improved COM velocity at $320 \mathrm{~ms}$. A significant increase in stabilising arm action was observed at $400 \mathrm{~ms}$, at exactly the same time observed for voluntary arm abduction (Grin et al. 2007). This phase of arm abduction was correlated with improvements in COM velocity at $540 \mathrm{~ms}$.

These actions of the arms and knees during voluntary trunk bending raises the question concerning which combination of voluntary motion at the arms, knees, and trunk would be an optimal strategy in patients prone to fall. This and our previous studies (Küng et al. 2009b; Grin et al. 2007) would suggest that a combined trunk and knee action would be most effective, as knee action provided early stabilisation both laterally and in the AP direction, and trunk motion brings greater lateral stabilisation overall. Furthermore, as we shall discuss below integration of the muscle responses into one synergy appears to work most effectively for both knee and trunk action.

One of the major issues concerning APAs and the subsequent change in posture associated with the main focal action be it to bend the trunk or flex one knee or laterally raise an arm concerns the extent to which these two types of muscle activity commands underlying these movements are programmed independently (Schepens and Drew 2003). Here, we have raised an additional aspect to the control synergies and associated motor commands. By adding the need for a balance correction we are asking a broader question, namely whether the automatic balance correcting synergy and later subsequent stabilising action can be programmed at the same time as the APA and later focal voluntary action and under which conditions. APAs cover a time period during which both stretch reflex and APRs activity of balance corrections occur. Further focal voluntary action and later (after $400 \mathrm{~ms}$ from tilt onset) balance stabilising action overlap when voluntary knee bending or trunk bending is performed. Thus, combined programming of the two commands is possible (Küng et al. 2009b; Grin et al. 2007). If both stretch reflex and APA contribute sensory information in planning APRs combined with later stabilising action, and focal voluntary action, then presumably the interactions among afferent information from stretch reflexes and APA could cause errors to occur when the APR, the stabilising action, and focal voluntary action should be simultaneously executed based on this information. Recent work has indicated that such interactions occur with stretch reflex gains (and presumably afferent feedback) being altered by APAs over a time course different from that of APA (Vedula et al. 2008). These interactions may underlie the instability of APRs when stretch reflexes and APAs have oppositely directed activity patterns (Oude Nijhuis et al. 2007). Thus, one of the prerequisites for integrating balance corrections and focal voluntary movements together would appear to be that sensory information provided by APA and stretch reflexes should be complementary. Here, we assume that afferent information underlying stretch reflex activity and resulting from APA is used for triggering and modulating APRs, later stabilising activity and focal voluntary movements respectively. Furthermore, we assume that this combined afferent information opens up supplementary interneuronal pathways enabling more effective recruitment of motor units. This action leads to greater than predicted biomechanical effects during balance corrections, and appears to permit maintaining balance with less muscle activity during later stabilising action.

Even when it is assumed that early sensory information provided by stretch reflexes is complementary to that of APA, the question still arises how the main motor programs are released. If the APA provides sensory information to release the main focal action, then both the APR and focal voluntary action will have been planned in a feedback and not as generally assumed in a feedforward mode for the focal action (Bouisset and Zattara 1981; Crenna and Frigo 1991).

The combined programming of focal voluntary with later stabilising activity action appears to be simpler for unilateral knee bending compared to bilateral knee bending as the timing of APA and APRs in knee muscles is very similar yet the focal voluntary action is very different from the later stabilising activity of balance corrections. In the current studies, focal voluntary action in trunk muscles consisted of a intermediate phase of activity between 200 and $400 \mathrm{~ms}$ not observed with voluntary knee bending. Nonetheless, this additional activity was well integrated into a combined action. The main signature of a successful integration of the balance stabilising and voluntary activity into one synergy appears to be that combined activity patterns should consist of activation with amplitudes less than those predicted from the individual actions. Presumably, CNS has less difficulty integrating motor programs together that overlap initially over the first $100 \mathrm{~ms}$ of the APR with 
subsequent phases consisting of excitatory activity patterns which do not overlap completely. Our working hypothesis would be that a voluntary response pattern which required a decrease in activity would not be well integrated into a combined activation pattern. However, it may well be that motor cortex programs are variable enough to take this combination into account too (Karayannidou et al. 2009).

Here, we have concentrated on cued commands for voluntary action at the initiation of the perturbation to stance. The question arises if the voluntary motor patterns are altered when the cue occurs before or after the perturbation. It remains for future research to determine the effect of variable cue timing on effective motor control for improving balance quite apart from any new insight into CNS motor programming such experiments could yield.

Acknowledgments This project was supported by a grant from the Swiss National Research Foundation (No. 320000-117950) to JHJ Allum.

\section{References}

Allum JHJ, Carpenter MG, Honegger F, Adkin AL, Bloem BR (2002) Age-dependent variations in the directional sensitivity of balance corrections and compensatory arm movements in man. J Physiol 542:643-663

Allum JHJ, Carpenter MG, Honegger F (2003) Directional Aspects of balance corrections in man. Employing multidirectional perturbations to better understand dynamic postural control in normal and balance-deficient populations. IEEE Eng Med Biol Mag 22:37-47

Allum JHJ, Oude Nijhuis LB, Carpenter MG (2008) Differences in coding provided by proprioceptive and vestibular sensory signals may contribute to lateral instability in vestibular loss subjects. Exp Brain Res 184:391-410

Bouisset S, Zattara M (1981) A sequence of postural movement precedes voluntary movement. Neurosci Lett 22:263-270

Carpenter MG, Allum JHJ, Honegger F (1999) Directional sensitivities of stretch reflexes and balance corrections for normal subjects in the roll and pitch planes. Exp Brain Res 129:93-113

Carpenter GM, Allum JHJ, Honegger F (2001) Vestibular influences on human postural control in combinations of pitch and roll planes reveal differences in spatiotemporal processing. Exp Brain Res 140:95-111

Carpenter MG, Allum JHJ, Honegger F, Adkin AL, Bloem BR (2004) Postural abnormalities to multi-directional stance perturbations in Parkinson's disease. J Neurol Neurosurg Psychiat 75:1245-1254

Crenna P, Frigo C (1991) A motor program for the initiation of forward-oriented movements in humans. J Physiol (London) 437:635-653

Davis JR, Carpenter MG, Tschanz R, Meyes S, Debrunner D, Burger J, Allum JHJ (2010) Trunk sway reduction in the young and elderly using multi-modal biofeedback to postural equilibrium. Gait Posture (in press)

Friedli WG, Hallett M, Simon SR (1984) Postural adjustments associated with rapid voluntary arm movements 1 . Electromyographic data. J Neurol Neurosurg Psychiatry 47:611-622

Grin L, Frank J, Allum JHJ (2007) The effect of voluntary arm abduction on balance recovery following multidirectional stance perturbations. Exp Brain Res 178:62-78
Hughey LK, Fung J (2005) Postural responses triggered by multidirectional leg lifts and surface tilts. Exp Brain Res 165:152-166

Karayannidou A, Bebozerova IN, Zelenin PV, Stout EE et al (2009) Activity of pyramidal tract neurons in the cat during standing and walking on an inclined plane. J Physiol (London) 587:37953811

Keshner EA, Allum JH, Pfaltz CR (1987) Postural coactivation and adaptation in the sway stabilizing responses of normals and patients with bilateral vestibular deficit. Exp Brain Res 69:77-92

Küng UM, Horlings GC, Honegger F, Kremer HPH, van de Warrenburg BPC, Bloem BR, Allum JHJ (2009a) Postural instability in cerebellar ataxia: Correlations with knee, arm and trunk movements. Neurosci 159:390-404

Küng UM, Horlings GC, Honegger F, Allum JHJ (2009b) Incorporating voluntary unilateral knee flexion into balance corrections elicited by multi-directional perturbations to stance. Neurosci 163:466-481

Küng UM, Horlings GC, Honegger F, Duysens JEJ, Allum JHJ (2009c) Control of roll and pitch motion during multi-directional balance perturbations. Exp Brain Res 194:631-645

Marigold DS, Eng JJ (2006) Altered timing of postural reflexes contributes to falling in persons with chronic stroke. Exp Brain Res 171:459-468

Marigold DS, Eng JJ, Dawson AS, Inglis JT, Harris JE, Gylfadottir S (2005) Exercise leads to faster postural reflexes, improved balance and mobility, and fewer falls in older persons with chronic stroke. J Am Geriatr Soc 53:416-423

Nashner LM, Cordo PJ (1981) Relation of automatic postural responses and reaction-time voluntary movements of human leg muscles. Exp Brain Res 43:395-405

Oude Nijhuis LB, Bloem BR, Carpenter MG, Allum JHJ (2007) Incorporating voluntary knee flexion into non-anticipatory balance corrections. J Neurophysiol 98:3047-3059

Oude Nijhuis LB, Allum JHJ, Borm GF, Honegger F, Overeem S, Bloem BR (2009) Directional sensitivity of "first-trial" motions in human balance control. J Neurophysiol 101:2802-2814

Pozzo T, Quamer M, Gentil C (2001) Simulating mechanical consequences of voluntary movement upon whole-body equilibrium: the arm raising paradigm revisited. Biol Cybern 85:39-49

Preuss R, Fung J (2008) Musculature and biomechanics of the trunk in the maintenance of upright posture. J Electromyogr Kinesiol $18: 815-828$

Schepens B, Drew T (2003) Strategies for the integration of posture and movement during reaching in the cat. $\mathrm{J}$ Neurophysiol 90:3066-3086

Vedula S, Stapley PJ, Kearney RE (2008) Reflex changes associated with anticipatory postural adjustments preceding voluntary arm movements in standing humans. Trans 30th Ann Int IEEE EMBS Conf 4523-4526

Verhoeff LL, Janssen LJF, Horlings CGC, Allum JHJ (2009) Effects of biofeedback on trunk sway during dual tasking in healthy young and elderly. Gait Posture 30:76-81

Visser JE, Allum JHJ, Carpenter MG, Esselink RA, Speelman JD, Borm GF, Bloem BR (2008) Subthalamic nucleus stimulation and levodopa-resistant postural instability in Parkinson's disease. J Neurol 255:205-210

Winter DA, Patla AE, Ishac M, Gage WH (2003) Motor mechanisms of balance during quiet standing. $\mathrm{J}$ Electromyogr Kinesiol 13:49-56

Wu G (1998) Age-related diefferences in body segmental movement during perturbed stance in humans. Clin Biomech 13:300-307 